\title{
Laguerre-Galerkin method for nonlinear partial differential equations on a semi-infinite interval
}

\author{
Ben-Yu Guo ${ }^{1, \star}$, Jie Shen ${ }^{2, \star \star}$ \\ 1 Department of Mathematics, Shanghai Normal University, Shanghai, 200234, China \\ 2 Department of Mathematics, Penn State University, University Park, PA16802, USA
}

Received October 6, 1997 / Revised version received July 22, 1999 /

Published online June 21, 2000 - (c) Springer-Verlag 2000

Summary. A Laguerre-Galerkin method is proposed and analyzed for the Burgers equation and Benjamin-Bona-Mahony (BBM) equation on a semiinfinite interval. By reformulating these equations with suitable functional transforms, it is shown that the Laguerre-Galerkin approximations are convergent on a semi-infinite interval with spectral accuracy. An efficient and accurate algorithm based on the Laguerre-Galerkin approximations to the transformed equations is developed and implemented. Numerical results indicating the high accuracy and effectiveness of this algorithm are presented.

Mathematics Subject Classification (1991): 65N35, 65N22, 65F05, 35J05

\section{Introduction}

While the Legendre- or Chebyshev-spectral approximations for PDEs in bounded domains have achieved great success and popularity in recent years (see e.g. $[11,8,5]$ ), spectral approximations for PDEs in unbounded domains using Laguerre polynomials have only received limited attention. Pioneer work on Laguerre approximation was developed in Gottlieb and Orszag, [11] and Maday, Pernaud-Thomas and Vandeven [15], see also Funaro [10]. In $[16,9,14]$ are numerical investigations to model linear elliptic equations

\footnotetext{
* The work of this author is patially supported by Chinese State Key project of Basic Research, N. 1999032804

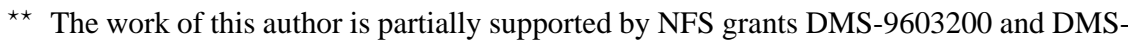
9706951

Correspondence to: $\mathrm{B}$. Guo
} 
using Laguerre-collocation or Laguerre-tau approximations, where difficulties associated with the extremely ill-conditioned behaviors of the Laguerrespectral approximations were reported. Furthermore, there seems to be no theoretical result available on Laguerre-spectral approximation for nonlinear PDEs. Here, in addition to the numerical difficulties mentioned above, another serious difficulty lies in the fact that the original formulations of many nonlinear PDEs of interest, e.g. Burgers equation, BBM equation, KDV equation and Kuramoto-Sivashinsky equation, are not well-posed in a weighted (with weight $\mathrm{e}^{-x}$ ) variational formulation on which Laguerrespectral approximations are often based. Thus, the key to construct a stable and convergent Laguerre-spectral approximation for such a nonlinear PDE is to find a suitable transform such that the weighted variational formulation of the transformed equation becomes well-posed.

The aim of this paper is to develop and analyze a Laguerre-Galerkin approximation and to construct an efficient, accurate and stable numerical algorithm for the Burgers equation and Benjamin-Bona-Mahony (BBM) equation on a semi-infinite interval.

The Burgers equation can be viewed as an one-dimensional model for the Navier-Stokes equations. Hence, solving the Burgers equation on a semiinfinite interval is a first step towards solving the Navier-Stokes equations in exterior domains which is an extremely challenging numerical task. The BBM equation was introduced in [4] to model the movement of regularized long waves. Numerous papers have been devoted to studying the BBM equation both analytically and numerically (see for instance $[6,1,3,7,13]$ ). However, most of the investigations were concerned with pure initial value problems or initial-boundary value problems. In many practical cases, e.g. water waves in a narrow and shallow stream coming from a large water reservoir or waves originated by a wave maker, we are also interested in studying the BBM equation on a semi-infinite interval.

For nonlinear PDEs on a semi-infinite interval, it may not be convenient to use finite-difference method or finite-element method, since we have to set up an artificial boundary and impose certain artificial boundary conditions which may contaminate the accuracy of approximate solutions. Since the Laguerre polynomials form an orthogonal system on the half line, it is natural to approximate nonlinear PDEs on a semi-infinite interval by using a Laguerre-spectral approximation. The techniques presented in this paper will be useful for studying other linear and nonlinear PDEs in fluid dynamics, quantum mechanics and other fields.

The rest of the paper is organized as follows. In Sect. 2, we provide some basic results on Laguerre approximations. In Sect. 3, we propose a suitable Laguerre-Galerkin approximation for the BBM equation and present the stability and error analysis. In Sect. 4, we consider the Burgers equation. In 
Sect. 5, we construct efficient algorithms for the Burgers equation and BBM equation, and present some numerical results. Some concluding remarks are given in the final section.

\section{Some preliminary results on Laguerre approximation}

The Laguerre polynomial of degree $l$ is defined by

$$
L_{l}(x)=\frac{1}{l !} \mathrm{e}^{x} \frac{d^{l}}{d x^{l}}\left(x^{l} \mathrm{e}^{-x}\right), \quad l=0,1,2, \ldots
$$

Note that $L_{l}(x)$ is the $l$-th eigenfunction of the singular Sturm-Liouville problem

$$
\frac{d}{d x}\left(x \mathrm{e}^{-x} \frac{d v(x)}{d x}\right)+\lambda \mathrm{e}^{-x} v(x)=0
$$

with the corresponding eigenvalue $\lambda_{l}=l$. For any $l \geq 0$, we have

$$
\left|L_{l}(x)\right| \leq \mathrm{e}^{\frac{x}{2}}, \quad x \in \mathbb{R}_{+} .
$$

Let us now define some suitable functional spaces for Laguerre approximation. Let $\omega(y)=\mathrm{e}^{-y}$ and

$$
L_{\omega}^{p}\left(\mathbb{R}_{+}\right)=\left\{v \mid\|v\|_{L_{\omega}^{p}}<\infty\right\}
$$

where

$$
\|v\|_{L_{\omega}^{p}}= \begin{cases}\left(\int_{\mathbb{R}_{+}}|v(y)|^{p} \omega(y) d y\right)^{\frac{1}{p}}, & \text { if } 1 \leq p<\infty \\ \operatorname{ess} \sup _{y \in \mathbb{R}_{+}}|v(y)|, & \text { if } p=\infty .\end{cases}
$$

In particular, $L_{\omega}^{2}\left(\mathbb{R}_{+}\right)$is a Hilbert space equipped with the following inner product and norm

$$
(u, v)_{\omega}=\int_{0}^{\infty} u(y) v(y) \omega(y) d y, \quad\|u\|_{\omega}=(u, u)_{\omega}^{\frac{1}{2}} .
$$

Furthermore, for any non-negative integer $m$, we define

$$
H_{\omega}^{m}\left(\mathbb{R}_{+}\right)=\left\{v \mid \frac{d^{k} v}{d x^{k}} \in L_{\omega}^{2}\left(\mathbb{R}_{+}\right), 0 \leq k \leq m\right\},
$$

which are associated with the following semi-norm and norm

$$
|v|_{m, \omega}=\left\|\frac{d^{m} v}{d x^{m}}\right\|_{\omega},\|v\|_{m, \omega}=\left(\sum_{k=0}^{m}|v|_{k, \omega}^{2}\right)^{\frac{1}{2}} .
$$


Then for any $r>0$, the space $H_{\omega}^{r}\left(\mathbb{R}_{+}\right)$and its norm $\|v\|_{r, \omega}$ can be defined by interpolation as in Adams [2]. We denote in particular

$$
H_{0, \omega}^{1}\left(\mathbb{R}_{+}\right)=\left\{v \mid v \in H_{\omega}^{1}\left(\mathbb{R}_{+}\right) \text {and } v(0)=0\right\} .
$$

It is well-known that

$$
\int_{0}^{\infty} L_{i}(x) L_{j}(x) \mathrm{e}^{-x} d x=\delta_{i j}, \quad \forall i, j \geq 0 .
$$

In other words, the set of Laguerre polynomials form an orthonormal basis for $L_{\omega}^{2}\left(\mathbb{R}_{+}\right)$, and any function $v \in L_{\omega}^{2}\left(\mathbb{R}_{+}\right)$can be expanded as

$$
v(x)=\sum_{l=0}^{\infty} \hat{v}_{l} L_{l}(x) \text { with } \hat{v}_{l}=\int_{\mathbb{R}_{+}} v(x) L_{l}(x) \mathrm{e}^{-x} d x .
$$

Let $N$ be any positive integer, and $\mathbb{P}_{N}$ be the space of polynomials of degree at most $N . \mathbb{P}_{N}^{0}=\mathbb{P}_{N} \cap H_{0, \omega}^{1}\left(\mathbb{R}_{+}\right)$. Let $P_{N}: L_{\omega}^{2}\left(\mathbb{R}_{+}\right) \rightarrow \mathbb{P}_{N}$ be the $L_{\omega}^{2}$-orthogonal projector, i.e., for any $v \in L_{\omega}^{2}\left(\mathbb{R}_{+}\right)$,

$$
\left(v-P_{N} v, \phi\right)_{\omega}=0, \quad \forall \phi \in \mathbb{P}_{N} .
$$

For any positive integer $\beta$, we define the space

$$
H_{\omega, \beta}^{r}\left(\mathbb{R}_{+}\right)=\left\{v \in H_{\omega}^{r}\left(\mathbb{R}_{+}\right) \mid x^{\frac{\beta}{2}} v \in H_{\omega}^{r}\left(\mathbb{R}_{+}\right)\right\}
$$

with the norm $\|v\|_{r, \omega, \beta}=\left\|v(1+x)^{\frac{\beta}{2}}\right\|_{r, \omega}$. As usual, $\omega$ will be omitted from the notations in case of $\omega \equiv 1$. Hereafter, $C$ will denote a generic positive constant independent of $N$.

Maday, Pernaud-Thomas and Vandeven [12] proved the following important result (see also [10] and [5]):

Lemma 2.1 Let $r \geq \mu \geq 0$ and $\beta$ be the largest integer for which $\beta<r+1$. Then

$$
\left\|v-P_{N} v\right\|_{\mu, \omega} \leq C N^{\mu-\frac{r}{2}}\|v\|_{r, \omega, \beta}, \quad \forall v \in H_{\omega, \beta}^{r}\left(\mathbb{R}_{+}\right) .
$$

We define the $H_{\omega}^{1}$-projector $P_{N}^{1}: H_{\omega}^{1}\left(\mathbb{R}_{+}\right) \rightarrow \mathbb{P}_{N}$ by $\left(\frac{d}{d x}\left(v-P_{N}^{1} v\right), \frac{d}{d x} \phi\right)_{\omega}+\left(v-P_{N}^{1} v, \phi\right)_{\omega}=0, \quad \forall \phi \in \mathbb{P}_{N}, v \in H_{\omega}^{1}\left(\mathbb{R}_{+}\right)$.

Similarly, we define the $H_{0, \omega}^{1}$-projector $P_{N}^{1,0}: H_{0, \omega}^{1}\left(\mathbb{R}_{+}\right) \rightarrow \mathbb{P}_{N}^{0}$ by

$$
\left(\frac{d}{d x}\left(v-P_{N}^{1,0} v\right), \frac{d}{d x} \phi\right)_{\omega}=0, \quad \forall \phi \in \mathbb{P}_{N}^{0}, v \in H_{0, \omega}^{1}\left(\mathbb{R}_{+}\right) .
$$




\section{Lemma 2.2}

$$
\begin{aligned}
\left\|\mathrm{e}^{-\frac{x}{2}} v\right\|_{L^{\infty}\left(\mathbb{R}_{+}\right)} & \leq \sqrt{2}\|v\|_{\omega}^{\frac{1}{2}}|v|_{1, \omega}^{\frac{1}{2}}, \quad \forall v \in H_{0, \omega}^{1}\left(\mathbb{R}_{+}\right) . \\
\|v\|_{\omega} & \leq 2|v|_{1, \omega},
\end{aligned}
$$

Proof. For any $x \in \mathbb{R}_{+}$,

$$
\begin{aligned}
\mathrm{e}^{-x} v^{2}(x) & =\int_{0}^{x} \frac{d}{d y}\left(\mathrm{e}^{-y} v^{2}(y)\right) d y \\
& =2 \int_{0}^{x} \mathrm{e}^{-y} v(y) \frac{d v(y)}{d y} d y-\int_{0}^{x} \mathrm{e}^{-y} v^{2}(y) d y,
\end{aligned}
$$

from which we derive

$$
\mathrm{e}^{-x} v^{2}(x)+\int_{0}^{x} \mathrm{e}^{-y} v^{2}(y) d y \leq 2 \int_{0}^{\infty} \mathrm{e}^{-y}\left|v(y) \frac{d v(y)}{d y}\right| d y \leq 2\|v\|_{\omega}|v|_{1, \omega},
$$

which implies the first conclusion. Letting $x \rightarrow \infty$, we get the second result.

Lemma 2.3 Let $r \geq 1$ and $\beta$ be the largest integer for which $\beta<r$. Then

$$
\left\|v-P_{N}^{1} v\right\|_{1, \omega} \leq C N^{\frac{1}{2}-\frac{r}{2}}\|v\|_{r, \omega, \beta}, \quad \forall v \in H_{\omega, \beta}^{r}\left(\mathbb{R}_{+}\right) .
$$

Proof. Let

$$
u(x)=v(0)+\int_{0}^{x} P_{N-1} \frac{d v(y)}{d y} d y .
$$

Then $v-u \in H_{0, \omega}^{1}\left(\mathbb{R}_{+}\right)$. Thanks to Lemma 2.1, we find

$$
\begin{aligned}
\left\|v-P_{N}^{1} v\right\|_{1, \omega} & \leq\|v-u\|_{1, \omega} \leq C|v-u|_{1, \omega}=\left\|\frac{d v}{d x}-P_{N-1} \frac{d v}{d x}\right\|_{\omega} \\
& \leq C N^{\frac{1}{2}-\frac{r}{2}}\left\|\frac{d v}{d x}\right\|_{r-1, \omega, \beta} \leq C N^{\frac{1}{2}-\frac{r}{2}}\|v\|_{r, \omega, \beta} .
\end{aligned}
$$

Lemma 2.4 Let $r \geq 1$ and $\beta$ be the largest integer for which $\beta<r$. Then

$$
\left\|v-P_{N}^{1,0} v\right\|_{1, \omega} \leq C N^{\frac{1}{2}-\frac{r}{2}}\|v\|_{r, \omega, \beta}, \quad \forall v \in H_{0, \omega}^{1}\left(\mathbb{R}_{+}\right) \cap H_{\omega, \beta}^{r}\left(\mathbb{R}_{+}\right) .
$$

Proof. By Lemma 2.2 and the definition of $P_{N}^{1,0}$,

$$
\begin{aligned}
\left\|v-P_{N}^{1,0} v\right\|_{1, \omega}^{2} & \leq C\left|v-P_{N}^{1,0} v\right|_{1, \omega}^{2}=C\left(\frac{d}{d x}\left(v-P_{N}^{1,0} v\right), \frac{d}{d x}(v-\phi)\right)_{\omega} \\
& \leq C\left\|v-P_{N}^{1,0} v\right\|_{1, \omega}|v-\phi|_{1, \omega}, \quad \forall \phi \in \mathbb{P}_{N}^{0} .
\end{aligned}
$$


Thus

$$
\left\|v-P_{N}^{1,0} v\right\|_{1, \omega} \leq C \inf _{\phi \in \mathcal{P}_{N}^{0}}|v-\phi|_{1, \omega} .
$$

Then, the desired result can be proved as in Lemma 2.3 by taking $\phi(x)=$ $\int_{0}^{x} P_{N-1} \frac{d v}{d y} d y$ in the above inequality.

\section{Laguerre-Galerkin method for the BBM equation}

The BBM equation (see [4]) on a semi-infinite interval reads:

$$
\left\{\begin{array}{l}
\partial_{t} v+\frac{1}{2} \partial_{y} v^{2}-\delta \partial_{t} \partial_{y}^{2} v=h, \quad y \in \mathbb{R}_{+}, t \in(0, T] \\
v(y, 0)=v_{0}(y), \quad y \in \mathbb{R}_{+}, \\
v(0, t)=g(t), \quad \lim _{y \rightarrow \infty} v(y, t)=\lim _{y \rightarrow \infty} \partial_{y} v(y, t)=0, \quad t \in(0, T]
\end{array}\right.
$$

where $T>0, \delta$ is a positive constant, $h(y, t)$ and $g(t)$ are respectively the source term and the boundary value at $y=0, v_{0}(y)$ is the initial value. We assume that the compatibility condition $v_{0}(0)=g(0)$ holds and that $h(y, t)$ and $v_{0}(y)$ decay fast enough when $y \rightarrow \infty$. The well-posedness of (3.1) in the classical sense can be found in [6,7].

We shall consider the Laguerre-Galerkin approximation of (3.1). Without loss of generality, we assume $g(t) \equiv 0$. In order to take advantage of the orthogonality of the Laguerre polynomials, it seems natural to consider the following weak formulation for (3.1):

Find $v \in L^{\infty}\left(0, T ; H_{0, \omega}^{1}\left(\mathbb{R}_{+}\right)\right)$such that

$$
\left\{\begin{array}{l}
\left(\partial_{t} v, \phi\right)_{\omega}+\frac{1}{2}\left(\partial_{y} v^{2}, \phi\right)_{\omega}+\delta\left(\partial_{t} \partial_{y} v, \partial_{y}(\phi \omega)\right)=(h, \phi)_{\omega}, \forall \phi \in H_{0, \omega}^{1}\left(\mathbb{R}_{+}\right), \\
v(\cdot, 0)=v_{0}(\cdot)
\end{array}\right.
$$

Unfortunately, this formulation is not suitable for the reasons specified below. In fact, taking $\phi=v$ in (3.2) and integrating by parts, we obtain

$$
\frac{1}{2} \frac{d}{d t}\left\{\|v\|_{\omega}^{2}+\delta|v|_{1, \omega}^{2}\right\}-\delta\left(\partial_{t} \partial_{y} v, v\right)_{\omega}=-\frac{1}{2}\left(\partial_{y}\left(v^{2}\right), v\right)_{\omega}+(h, v)_{\omega} .
$$

Notice that the last term on the left-hand side may not be positive and can not be properly controlled. Furthermore, as explained in Sect. 2, even in the absence of the nonlinear term, the above formulation will not lead to satisfactory approximation for $x$ large. Thus, we shall consider an alternative 
formulation of (3.1). We first make a change of variable: $y=\frac{\sqrt{\delta}}{2} x$, and let $w(x, t)=v(y, t), \tilde{h}(x, t)=h(y, t)$. Then, (3.1) becomes

$$
\partial_{t} w+\frac{1}{\sqrt{\delta}} \partial_{x}\left(w^{2}\right)-4 \partial_{t} \partial_{x}^{2} w=\tilde{h} .
$$

Setting

$$
\begin{aligned}
& u(x, t)=\mathrm{e}^{\frac{x}{2}} w(x, t)=\mathrm{e}^{\frac{x}{2}} v\left(\frac{\sqrt{\delta}}{2} x, t\right), \\
& f(x, t)=\frac{1}{4} \mathrm{e}^{\frac{x}{2}} \tilde{h}(x, t)=\frac{1}{4} \mathrm{e}^{\frac{x}{2}} h\left(\frac{\sqrt{\delta}}{2} x, t\right),
\end{aligned}
$$

the BBM equation (3.1) with $g(t) \equiv 0$ becomes

$$
\left\{\begin{array}{l}
\frac{1}{4 \sqrt{\delta}} \mathrm{e}^{\frac{x}{2}} \partial_{x}\left(\mathrm{e}^{-x} u^{2}\right)+\partial_{t} \partial_{x} u-\partial_{t} \partial_{x}^{2} u=f, \quad x \in \mathbb{R}_{+}, t \in(0, T] \\
u(x, 0)=u_{0}(x)=\mathrm{e}^{\frac{x}{2}} v_{0}\left(\frac{\sqrt{\delta}}{2} x\right) \\
u(0, t)=0, t \in(0, T] .
\end{array}\right.
$$

Let us denote

$$
\begin{aligned}
a(u, v) & =\int_{0}^{\infty} \frac{d u}{d x} \frac{d v}{d x} \mathrm{e}^{-x} d x, \\
b(u, z, v) & =b_{1}(u, z, v)+b_{2}(u, z, v),
\end{aligned}
$$

with

$$
\begin{aligned}
& b_{1}(u, z, v)=-\frac{1}{4} \int_{0}^{\infty} \mathrm{e}^{-\frac{3 x}{2}} u(x) z(x) \frac{d v}{d x} d x, \\
& b_{2}(u, z, v)=\frac{1}{8} \int_{0}^{\infty} \mathrm{e}^{-\frac{3 x}{2}} u(x) z(x) v(x) d x .
\end{aligned}
$$

The key property which imply the suitability of (3.3) is

\section{Lemma 3.1}

$$
b(u, u, u)=0, \quad \forall u \in H_{0, \omega}^{1}\left(\mathbb{R}_{+}\right) .
$$

Proof. The result follows from the definition and by integration by parts, since

$$
\begin{aligned}
b_{1}(u, u, u) & =-\frac{1}{4} \int_{0}^{\infty} \mathrm{e}^{-\frac{3 x}{2}} u^{2} \frac{d u}{d x} d x=-\frac{1}{12} \int_{0}^{\infty} \mathrm{e}^{-\frac{3 x}{2}} d u^{3} \\
& =-\frac{1}{8} \int_{0}^{\infty} \mathrm{e}^{-\frac{3 x}{2}} u^{3} d x=-b_{2}(u, u, u), \quad \forall u \in H_{0, \omega}^{1}\left(\mathbb{R}_{+}\right) .
\end{aligned}
$$


Hence, we shall consider the following variational formulation of (3.3): find $u \in L^{\infty}\left(0, T ; H_{0, \omega}^{1}\left(\mathbb{R}_{+}\right)\right)$such that

$$
\left\{\begin{array}{l}
\frac{1}{\sqrt{\delta}} b(u(t), u(t), \phi)+a\left(\partial_{t} u(t), \phi\right)=(f(t), \phi)_{\omega}, \\
\quad \forall \phi \in H_{0, \omega}^{1}\left(\mathbb{R}_{+}\right), t \in(0, T] \\
u(0)=u_{0} .
\end{array}\right.
$$

The Laguerre-Galerkin approximation for (3.6) is: find $u_{N}(t) \in \mathbb{P}_{N}^{0}$ for all $0 \leq t \leq T$, such that

$$
\left\{\begin{array}{c}
\frac{1}{\sqrt{\delta}} b\left(u_{N}(t), u_{N}(t), \phi\right)+a\left(\partial_{t} u_{N}(t), \phi\right)=(f, \phi)_{\omega}, \\
\forall \phi \in \mathbb{P}_{N}^{0}, t \in(0, T], \\
u_{N}(0)=u_{N, 0}=P_{N}^{1,0} u_{0} .
\end{array}\right.
$$

Let us first establish the following result:

Lemma 3.2 Let $f \in L^{2}\left(0, T ; L_{\omega}^{2}\left(\mathbb{R}_{+}\right)\right)$and $u_{0} \in H_{0, \omega}^{1}\left(\mathbb{R}_{+}\right)$. Let $u$ and $u_{N}$ be respectively the solution of (3.6) and (3.7). Then

$$
\|u(t)\|_{1, \omega},\left\|u_{N}(t)\right\|_{1, \omega} \leq C\left(\|f\|_{L^{2}\left(0, T ; L_{\omega}^{2}\left(\mathbb{R}_{+}\right)\right)}+\left\|u_{0}\right\|_{1, \omega}\right), \quad t \in(0, T] .
$$

Proof. Take $\phi=u(t)$ in (3.6), thanks to Lemmas 3.1 and 2.2, we find

$$
\frac{d}{d t}|u(t)|_{1, \omega}^{2}=2(f(t), u(t))_{\omega} \leq 2\|f(t)\|_{\omega}\|u(t)\|_{\omega} \leq C\|f(t)\|_{\omega}|u(t)|_{1, \omega} .
$$

Applying the Gronwall lemma to the above inequality, we obtain the desired result for $u(t)$. The result for $u_{N}(t)$ can be proved similarly.

Hereafter, we use $C_{1}$ to denote a generic positive constant which may depend on the data (i.e. $u_{0}, f, r$ ), but is independent of $N$. We now analyze the stability of (3.7) in the sense of Guo [12,13] and Stetter [19].

Theorem 3.1 Let $u_{N}$ and $\hat{u}_{N}$ be respectively the solutions of (3.7) with data $\left(f, u_{N, 0}\right)$ and $\left(\hat{f}, \hat{u}_{N, 0}\right)$. We denote $\tilde{f}=\hat{f}-f$ and $\tilde{u}_{N}=\hat{u}_{N}-u_{N}$. Then

$$
\left|\tilde{u}_{N}(t)\right|_{1, \omega}^{2} \leq C_{1}\left(\left|\tilde{u}_{N, 0}\right|_{1, \omega}^{2}+\int_{0}^{\mathrm{t}}\|\tilde{f}(s)\|_{\omega}^{2} d s\right), \quad \forall t \in(0, T] .
$$

Proof. By definition in (3.4-3.5), we have

$$
b(u, v, \phi)=b(v, u, \phi), \quad \forall u, v, \phi \in H_{0, \omega}^{1}\left(\mathbb{R}_{+}\right) .
$$


Hence, $\tilde{u}_{N}$ satisfies the following equation:

$$
\left\{\begin{aligned}
\frac{1}{\sqrt{\delta}} b\left(\tilde{u}_{N}(t), \tilde{u}_{N}(t), \phi\right) & +\frac{2}{\sqrt{\delta}} b\left(\tilde{u}_{N}(t), u_{N}(t), \phi\right) \\
& +a\left(\partial_{t} \tilde{u}_{N}(t), \phi\right)=(\tilde{f}(t), \phi)_{\omega}, \forall \phi \in \mathbb{P}_{N}^{0} \\
\tilde{u}_{N}(0)=\tilde{u}_{N, 0} . &
\end{aligned}\right.
$$

By taking $\phi=2 \tilde{u}_{N}$ in (3.9), it follows from Lemma 3.1 that

$$
\frac{d}{d t}\left|\tilde{u}_{N}(t)\right|_{1, \omega}^{2}+\frac{4}{\sqrt{\delta}} b\left(\tilde{u}_{N}(t), u_{N}(t), \tilde{u}_{N}(t)\right) \leq 2\|\tilde{f}(t)\|_{\omega}^{2}+\left|\tilde{u}_{N}(t)\right|_{1, \omega}^{2} .
$$

Thanks to Lemma 2.2, we find

$$
\begin{aligned}
4\left|b_{1}\left(\tilde{u}_{N}(t), u_{N}(t), \tilde{u}_{N}(t)\right)\right| & \leq\left\|\mathrm{e}^{-\frac{x}{2}} u_{N}(t)\right\| L_{L^{\infty}}\left\|\tilde{u}_{N}(t)\right\|_{\omega}\left|\tilde{u}_{N}(t)\right|_{1, \omega} \\
& \leq C_{1}\left\|\tilde{u}_{N}(t)\right\| \|_{\omega}\left|\tilde{u}_{N}(t)\right|_{1, \omega} \leq C_{1}\left|\tilde{u}_{N}(t)\right|_{1, \omega}^{2}
\end{aligned}
$$

Similarly, we have

$$
4\left|b_{2}\left(\tilde{u}_{N}(t), u_{N}(t), \tilde{u}_{N}(t)\right)\right| \leq C_{1}\left\|\tilde{u}_{N}(t)\right\|_{\omega}^{2} \leq C_{1}\left|\tilde{u}_{N}(t)\right|_{1, \omega}^{2} .
$$

Thanks to the above estimates, we find that

$$
\frac{d}{d t}\left|\tilde{u}_{N}(t)\right|_{1, \omega}^{2} \leq C_{1}\left(\|\tilde{f}(t)\|_{\omega}^{2}+\left|\tilde{u}_{N}(t)\right|_{1, \omega}^{2}\right) .
$$

We conclude by applying the Gronwall lemma to the above inequality.

We now consider the error analysis for (3.7). Let $w_{N}=P_{N}^{1,0} u$. Then it follows from (3.6) that

$$
\left\{\begin{array}{l}
\frac{1}{\sqrt{\delta}} b\left(w_{N}(t), w_{N}(t), \phi\right)+a\left(\partial_{t} w_{N}(t), \phi\right) \\
\quad+e\left(u(t), w_{N}(t), \phi\right)=(f(t), \phi)_{\omega}, \quad \forall \phi \in \mathbb{P}_{N}^{0}, \\
w_{N}(0)=P_{N}^{1,0} u_{0}
\end{array}\right.
$$

where

$$
e\left(u(t), w_{N}(t), \phi\right)=b(u(t), u(t), \phi)-b\left(w_{N}(t), w_{N}(t), \phi\right) .
$$

Let us denote $\tilde{u}_{N}(t)=u_{N}(t)-w_{N}(t)$ and subtract (3.11) from (3.7), we obtain that

$$
\left\{\begin{array}{l}
\frac{1}{\sqrt{\delta}} b\left(\tilde{u}_{N}(t), \tilde{u}_{N}(t), \phi\right)+\frac{2}{\sqrt{\delta}} b\left(\tilde{u}_{N}(t), w_{N}(t), \phi\right) \\
\quad+a\left(\partial_{t} \tilde{u}_{N}(t), \phi\right)=e\left(u(t), w_{N}(t), \phi\right), \forall \phi \in \mathbb{P}_{N}^{0} \\
\tilde{u}_{N}(0)=\tilde{u}_{N, 0}=0 .
\end{array}\right.
$$


Theorem 3.2 Let $u$ and $u_{N}$ be respectively the solution of (3.3) and (3.7). Let $r \geq 1$ and $\beta$ be the largest integer for which $\beta<r$. Then

$$
\begin{aligned}
& \left\|u_{N}(t)-u(t)\right\|_{1, \omega} \leq C_{1} N^{\frac{1}{2}-\frac{r}{2}}\left(\left\|u_{0}\right\|_{r, \omega, \beta}+\|u\|_{L^{2}\left(0, T ; H_{\omega, \beta}^{r}\left(\mathbb{R}_{+}\right)\right)}\right), \\
& \quad \forall t \in[0, T] .
\end{aligned}
$$

Proof. Letting $\phi=2 \tilde{u}_{N}(t)$ in (3.12), thanks to Lemma 3.1, we find

$$
\frac{d}{d t}\left|\tilde{u}_{N}(t)\right|_{1, \omega}^{2}=2 e\left(u(t), w_{N}(t), \tilde{u}_{N}(t)\right)-\frac{4}{\sqrt{\delta}} b\left(\tilde{u}_{N}(t), w_{N}(t), \tilde{u}_{N}(t)\right) .
$$

In virtue of Lemmas 2.2 and 3.2, we have

$$
\left|4 b\left(\tilde{u}_{N}(t), w_{N}(t), \tilde{u}_{N}(t)\right)\right| \leq C_{1}\left|\tilde{u}_{N}(t)\right|_{1, \omega}^{2},
$$

and

$$
\begin{aligned}
&\left|b_{1}\left(u(t), u(t), \tilde{u}_{N}(t)\right)-b_{1}\left(w_{N}(t), w_{N}(t), \tilde{u}_{N}(t)\right)\right| \\
&=\frac{1}{4}\left|\int_{0}^{\infty} \mathrm{e}^{-\frac{3 x}{2}}\left(u(t)+w_{N}(t)\right)\left(u(t)-w_{N}(t)\right) \partial_{x} \tilde{u}_{N}(t) d x\right| \\
& \leq \frac{\sqrt{2}}{4}\left\|u(t)+w_{N}(t)\right\|_{\omega}^{\frac{1}{2}}\left|u(t)+w_{N}(t)\right|_{1, \omega}^{\frac{1}{2}} \\
& \\
& \leq \frac{1}{8}\left|\tilde{u}_{N}(t)\right|_{1, \omega}^{2}+\sqrt{2}\left|u(t)+w_{N}(t)\right|_{1, \omega}^{2}\left\|u(t)-w_{N}(t)\right\|_{\omega}^{2} \\
& \leq \frac{1}{8}\left|\tilde{u}_{N}(t)\right|_{1, \omega}^{2}+C N^{1-r}|u(t)|_{1, \omega}^{2}\|u(t)\|_{r, \omega, \beta}^{2} .
\end{aligned}
$$

Similarly, we can estimate the term

$$
\left|b_{2}\left(u(t), u(t), \tilde{u}_{N}(t)\right)-b_{2}\left(w_{N}(t), w_{N}(t), \tilde{u}_{N}(t)\right)\right| .
$$

The theorem is then a consequence of the above inequalities and Gronwall lemma.

We can now derive an error estimate for the approximation of the original BBM equation (3.1).

Theorem 3.3 Let $v_{N}(y, t)=\mathrm{e}^{-\frac{y}{\sqrt{\delta}}} u_{N}\left(\frac{2 y}{\sqrt{\delta}}, t\right)$ be the approximation of $v(y, t)$, the original solution of (3.1). Then

$$
\left\|v_{N}(t)-v(t)\right\|_{1} \leq C_{1} N^{\frac{1}{2}-\frac{r}{2}}\|v\|_{L^{\infty}\left(0, T ; H_{\beta}^{r}\left(\mathbb{R}_{+}\right)\right)}, \quad \forall t \in[0, T] .
$$


Proof. Since $\left(v-v_{N}\right)=\mathrm{e}^{-x / 2}\left(u-u_{N}\right)$, we find

$$
\partial_{y}\left(v-v_{N}\right)=\frac{2}{\sqrt{\delta}} \mathrm{e}^{-x / 2}\left(\partial_{x}\left(u-u_{N}\right)-\frac{1}{2}\left(u-u_{N}\right)\right) .
$$

Hence

$$
\left\|\left(v-v_{N}\right)(t)\right\|_{1} \leq C\left\|u-u_{N}(t)\right\|_{1, \omega} \leq C N^{\frac{1}{2}-\frac{r}{2}}\|u(t)\|_{r, \omega, \beta} .
$$

We conclude from the above and the fact that $\|u(t)\|_{r, \omega, \beta} \leq C\|v(t)\|_{r, \beta}$ which can be proved straightforwardly.

Remark 3.1 The above result indicates that the Laguerre-Galerkin approximation may converge exponentially, even if $v$ only delays algebraicly at infinity, as long as $\|v\|_{L^{\infty}\left(0, T ; H_{\beta}^{r}\left(\mathbb{R}_{+}\right)\right)}$are finite for all $r>0$ (see numerical results in Sect. 5).

\section{Laguerre-Galerkin method for the Burgers equation}

The Burgers equation on a semi-infinite interval reads:

$$
\left\{\begin{array}{l}
\partial_{t} v+\frac{1}{2} \partial_{y} v^{2}-\nu \partial_{y}^{2} v=h, \quad y \in \mathbb{R}_{+}, t \in(0, T] \\
v(y, 0)=v_{0}(y), \quad y \in \mathbb{R}_{+}, \\
v(0, t)=0, \quad \lim _{y \rightarrow \infty} v(y, t)=\lim _{y \rightarrow \infty} \partial_{y} v(y, t)=0, \quad t \in(0, T]
\end{array}\right.
$$

where $\nu$ is a positive constant. We assume a homogeneous boundary condition without loss of generality. As for the BBM equation, the original Burgers equation is not suitable for the Laguerre approximation. Hence, similarly as in the previous section, we set

$$
u(x, t)=\mathrm{e}^{\frac{x}{2}} v(x, t), \quad f(x, t)=\mathrm{e}^{\frac{x}{2}} h(x, t) .
$$

Therefore, (4.1) becomes

$$
\left\{\begin{array}{l}
\partial_{t} u-\nu\left(\partial_{x}^{2} u-\partial_{x} u+\frac{1}{4} u\right)+\frac{1}{2} \mathrm{e}^{\frac{x}{2}} \partial_{x}\left(\mathrm{e}^{-x} u^{2}\right)=f \\
\quad x \in \mathbb{R}_{+}, t \in(0, T] \\
u(x, 0)=u_{0}(x)=\mathrm{e}^{\frac{x}{2}} v_{0}(x) \\
u(0, t)=0, t \in(0, T] .
\end{array}\right.
$$

Let us denote

$$
\begin{aligned}
a(u, v) & =\int_{0}^{\infty}\left(\frac{d u}{d x} \frac{d v}{d x}-\frac{1}{4} u v\right) \mathrm{e}^{-x} d x, \\
b(u, z, v) & =2\left(b_{1}(u, z, v)+b_{2}(u, z, v)\right),
\end{aligned}
$$


where $b_{1}$ and $b_{2}$ are defined in (3.5). We recall from Lemma 3.1 that

$$
b(u, u, u)=0, \quad \forall u \in H_{0, \omega}^{1}\left(\mathbb{R}_{+}\right) .
$$

We also derive from Lemma 2.2 that

$$
a(u, u)=|u|_{1, \omega}^{2}-\frac{1}{4}\|u\|_{\omega}^{2} \geq 0, \forall u \in H_{0, \omega}^{1}\left(\mathbb{R}_{+}\right) .
$$

A variational formulation for (4.2) is:

find $u \in L^{\infty}\left(0, T ; H_{0, \omega}^{1}\left(\mathbb{R}_{+}\right)\right)$such that

$$
\left\{\begin{array}{r}
\left(\partial_{t} u(t), \phi\right)_{\omega}+\nu a(u(t), \phi) \\
+b(u(t), u(t), \phi)=(f(t), \phi)_{\omega}, \\
\quad \forall \phi \in H_{0, \omega}^{1}\left(\mathbb{R}_{+}\right), t \in(0, T], \\
u(0)=u_{0} .
\end{array}\right.
$$

Its Laguerre-Galerkin approximation is:

find $u_{N}(t) \in \mathbb{P}_{N}^{0}$ for all $0 \leq t \leq T$, such that

$$
\left\{\begin{aligned}
\left(\partial_{t} u_{N}(t), \phi\right)_{\omega}+ & \nu a\left(u_{N}(t), \phi\right) \\
& +b\left(u_{N}(t), u_{N}(t), \phi\right)=(f(t), \phi)_{\omega} \\
\forall v \in \mathbb{P}_{N}^{0}, t \in(0, T] & \\
u_{N}(0)=u_{N, 0}= & P_{N} u_{0}
\end{aligned}\right.
$$

Lemma 4.1 Let $f \in L^{2}\left(0, T ; L_{\omega}^{2}\left(\mathbb{R}_{+}\right)\right)$and $u_{0} \in L_{\omega}^{2}\left(\mathbb{R}_{+}\right)$. Let $u$ and $u_{N}$ be respectively the solution of (4.6) and (4.7). Then

$$
\|u(t)\|_{\omega},\left\|u_{N}(t)\right\|_{\omega} \leq C\left(\|f\|_{L^{2}\left(0, T ; L_{\omega}^{2}\left(\mathbb{R}_{+}\right)\right)}+\left\|u_{0}\right\|_{\omega}\right), \quad t \in(0, T] .
$$

Proof. Take $\phi=2 u$ in (4.6), thanks to (4.3-4.4), we find

$$
\frac{d}{d t}\|u(t)\|_{\omega}^{2} \leq 2(f(t), u(t))_{\omega} \leq\|f(t)\|_{\omega}^{2}+\|u(t)\|_{\omega}^{2} .
$$

Applying the Gronwall lemma to the above inequality, we obtain the desired result for $u(t)$. The result for $u_{N}(t)$ can be proved similarly.

Theorem 4.1 Let $u_{N}$ and $\hat{u}_{N}$ be respectively the solutions of (4.7) with data $\left(f, u_{N, 0}\right)$ and $\left(\hat{f}, \hat{u}_{N, 0}\right)$. We denote $\tilde{f}=\hat{f}-f$ and $\tilde{u}_{N}=\hat{u}_{N}-u_{N}$. Then

$$
\left\|\tilde{u}_{N}(t)\right\|_{\omega}^{2} \leq C_{1}\left(\left\|\tilde{u}_{N, 0}\right\|_{\omega}^{2}+\int_{0}^{\mathrm{t}}\|\tilde{f}(s)\|_{\omega}^{2} d s\right), \quad \forall t \in(0, T] .
$$


Proof. Thanks to (3.8), we find that $\tilde{u}_{N}$ satisfies the following equation:

$$
\left\{\begin{array}{l}
\left(\partial_{t} \tilde{u}_{N}(t), \phi\right)+\nu a\left(\tilde{u}_{N}(t), \phi\right)+b\left(\tilde{u}_{N}(t), \tilde{u}_{N}(t), \phi\right) \\
\quad+2 b\left(\tilde{u}_{N}(t), u_{N}(t), \phi\right)=(\tilde{f}(t), \phi)_{\omega}, \forall \phi \in \mathbb{P}_{N}^{0}, \\
\tilde{u}_{N}(0)=\tilde{u}_{N, 0} .
\end{array}\right.
$$

By taking $\phi=2 \tilde{u}_{N}$ in (4.9), it follows from (4.4) that

$$
\begin{aligned}
\frac{d}{d t}\left|\tilde{u}_{N}(t)\right|_{\omega}^{2} & +2 \nu\left(\left|\tilde{u}_{N}(t)\right|_{1, \omega}^{2}-\frac{1}{4}\left\|\tilde{u}_{N}(t)\right\|_{\omega}^{2}\right) \\
& +4 b\left(\tilde{u}_{N}(t), u_{N}(t), \tilde{u}_{N}(t)\right) \leq\|\tilde{f}(t)\|_{\omega}^{2}+\left\|\tilde{u}_{N}(t)\right\|_{\omega}^{2} .
\end{aligned}
$$

Thanks to Lemmas 2.2, 4.1 and Young's inequality, we have

$$
\begin{aligned}
4\left|b_{1}\left(\tilde{u}_{N}(t), u_{N}(t), \tilde{u}_{N}(t)\right)\right| & \leq C\left\|\mathrm{e}^{-\frac{x}{2}} u_{N}(t)\right\|_{L^{\infty}\left(\mathbb{R}_{+}\right)}\left\|\tilde{u}_{N}(t)\right\|_{\omega}\left|\tilde{u}_{N}(t)\right|_{1, \omega} \\
& \leq C_{1}\left\|\tilde{u}_{N}(t)\right\| \|_{\omega}^{\frac{1}{2}}\left|\tilde{u}_{N}(t)\right|_{1, \omega}^{\frac{3}{2}} \\
& \leq \frac{\nu}{2}\left|\tilde{u}_{N}(t)\right|_{1, \omega}^{2}+C_{1}\left\|\tilde{u}_{N}(t)\right\|_{\omega}^{2} .
\end{aligned}
$$

Similarly, we have

$$
4\left|b_{2}\left(\tilde{u}_{N}(t), u_{N}(t), \tilde{u}_{N}(t)\right)\right| \leq \frac{\nu}{2}\left|\tilde{u}_{N}(t)\right|_{1, \omega}^{2}+C\left\|\tilde{u}_{N}(t)\right\|_{\omega}^{2} .
$$

Thanks to the above estimates, we find that

$$
\frac{d}{d t}\left|\tilde{u}_{N}(t)\right|_{\omega}^{2}+\nu\left|\tilde{u}_{N}(t)\right|_{1, \omega}^{2} \leq C_{1}\left(\|\tilde{f}(t)\|_{\omega}^{2}+\left\|\tilde{u}_{N}(t)\right\|_{\omega}^{2}\right) .
$$

We conclude by applying the Gronwall lemma to the above inequality.

Theorem 4.2 Let $u$ and $u_{N}$ be respectively the solution of (4.2) and (4.7). Let $s, r \geq 1, \alpha$ and $\beta$, be the largest integers for which $\alpha<s$ and $\beta<r$. Then,

$$
\begin{aligned}
\left\|u_{N}(t)-u(t)\right\|_{1, \omega} & \leq C_{1}\left(N^{\frac{1}{2}-\frac{s}{2}}\|u\|_{L^{\infty}\left(0, T ; H_{\omega, \alpha}^{s}\right.}\left(\mathbb{R}_{+}\right)\right) \\
& \left.+N^{\frac{1}{2}-\frac{r}{2}}\left\|u_{t}\right\|_{L^{2}\left(0, T ; H_{\omega, \beta}^{r}\left(\mathbb{R}_{+}\right)\right)}\right), \forall t \in(0, T] .
\end{aligned}
$$

Proof. Let $w_{N}=P_{N}^{1,0} u$ and we denote

$$
e_{N}=u-u_{N}=\left(u-w_{N}\right)+\left(w_{N}-u_{N}\right)=\xi_{N}+\eta_{N} .
$$

From Lemmas 2.2 and 2.4, we have

$$
\left\|\xi_{N}\right\|_{\omega} \leq 2\left|\xi_{N}\right|_{1, \omega} \leq C N^{\frac{1}{2}-\frac{s}{2}}\|u\|_{s, \omega, \alpha} .
$$


Subtracting (4.7) from (4.6), we get

$$
\begin{aligned}
\left(\partial_{t} e_{N}, v\right)_{\omega}+\nu a\left(e_{N}, v\right) & =b\left(u_{N}, u_{N}, v\right)-b(u, u, v) \\
& =-b\left(u_{N}, e_{N}, v\right)-b\left(e_{N}, u, v\right), \forall v \in \mathbb{P}_{N}^{0}
\end{aligned}
$$

Taking $v=\eta_{N}$ in the above relation, we obtain

$$
\begin{aligned}
\frac{1}{2} \frac{d}{d t}\left\|\eta_{N}\right\|_{\omega}^{2}+\nu\left(\left|\eta_{N}\right|_{1, \omega}^{2}-\frac{1}{4}\left\|\eta_{N}\right\|_{\omega}^{2}\right) & =-\left(\partial_{t} \xi_{N}, \eta_{N}\right)_{\omega}-\nu a\left(\xi_{N}, \eta_{N}\right) \\
& -b\left(u_{N}, e_{N}, \eta_{N}\right)-b\left(e_{N}, u, \eta_{N}\right)
\end{aligned}
$$

The terms on the right-hand side of (4.12) can be bounded as follows:

$$
\begin{gathered}
-\left(\partial_{t} \xi_{N}, \eta_{N}\right)_{\omega} \leq \frac{1}{2}\left(\left\|\partial_{t} \xi_{N}\right\|_{\omega}^{2}+\left\|\eta_{N}\right\|_{\omega}^{2}\right) \\
-a\left(\xi_{N}, \eta_{N}\right) \leq \frac{\nu}{4}\left|\xi_{N}\right|_{1, \omega}^{2}+C\left\|\eta_{N}\right\|_{1, \omega}^{2} .
\end{gathered}
$$

Using Lemmas 2.2, 4.1 and Young's inequality repeatedly, we have

$$
\begin{aligned}
b_{1}\left(u_{N}, e_{N}, \eta_{N}\right) & \leq C\left\|\mathrm{e}^{-\frac{x}{2}} e_{N}\right\|_{L^{\infty}\left(\mathbb{R}_{+}\right)}\left|\eta_{N}\right|_{1, \omega}\left\|u_{N}\right\|_{\omega} \\
& \leq C_{1}\left\|e_{N}\right\|_{\omega}^{\frac{1}{2}}\left|e_{N}\right|_{1, \omega}^{\frac{1}{2}}\left|\eta_{N}\right|_{1, \omega} \\
& \leq C_{1}\left(\left\|\xi_{N}\right\|_{\omega}^{\frac{1}{2}}+\left\|\eta_{N}\right\|_{\omega}^{\frac{1}{2}}\right)\left(\left|\xi_{N}\right|_{1, \omega}^{\frac{1}{2}}+\left|\eta_{N}\right|_{1, \omega}^{\frac{1}{2}}\right)\left|\eta_{N}\right|_{1, \omega} \\
& \leq \frac{\nu}{8}\left|\eta_{N}\right|_{1, \omega}^{2}+C_{1}\left(\left\|\eta_{N}\right\|_{\omega}^{2}+\left\|\xi_{N}\right\|_{1, \omega}^{2}\right) .
\end{aligned}
$$

The terms $b_{2}\left(u_{N}, e_{N}, \eta_{N}\right)$ and $b\left(e_{N}, u, \eta_{N}\right)$ can be bounded similarly and relatively easily. Combining all the above inequalities, we arrive at

$$
\frac{d}{d t}\left\|\eta_{N}\right\|_{\omega}^{2}+\nu\left|\eta_{N}\right|_{1, \omega}^{2} \leq C_{1}\left(\left\|\eta_{N}\right\|_{\omega}^{2}+\left\|\xi_{N}\right\|_{1, \omega}^{2}+\left\|\partial_{t} \xi_{N}\right\|_{\omega}^{2}\right)
$$

Therefore, we derive by applying Gronwall lemma that for all $t \in(0, T]$,

$$
\begin{aligned}
& \left\|\eta_{N}(t)\right\|_{\omega}^{2}+\nu \int_{0}^{\mathrm{t}}\left|\eta_{N}(s)\right|_{1, \omega}^{2} d s \\
& \quad \leq C_{1}\left(\left\|\eta_{N}(0)\right\|_{\omega}^{2}+\int_{0}^{\mathrm{t}}\left(\left\|\xi_{N}(s)\right\|_{1, \omega}^{2}+\left\|\partial_{s} \xi_{N}(s)\right\|_{\omega}^{2}\right) d s\right) .
\end{aligned}
$$


Hence, we derive from the above and (4.10) that for all $t \in(0, T]$,

$$
\begin{aligned}
\left\|u-u_{N}(t)\right\|_{\omega}^{2}+ & \nu \int_{0}^{\mathrm{t}}\left|u-u_{N}(s)\right|_{1, \omega}^{2} d s \\
\leq & C_{1}\left(\left\|\xi_{N}(0)\right\|_{\omega}^{2}+\left\|\xi_{N}(t)\right\|_{\omega}^{2}\right. \\
& \left.+\int_{0}^{\mathrm{t}}\left(\left\|\xi_{N}(s)\right\|_{1, \omega}^{2}+\left\|\partial_{s} \xi_{N}(s)\right\|_{\omega}^{2}\right) d s\right) \\
\leq & C_{1}\left(N^{\frac{1}{2}-\frac{s}{2}}\|u\|_{L^{\infty}\left(0, T ; H_{\omega, \alpha}^{s}\left(\mathbb{R}_{+}\right)\right)}\right. \\
& \left.+N^{\frac{1}{2}-\frac{r}{2}}\left\|u_{t}\right\|_{L^{2}\left(0, T ; H_{\omega, \beta}^{r}\left(\mathbb{R}_{+}\right)\right)}\right) .
\end{aligned}
$$

Now, let $v_{N}=\mathrm{e}^{-\frac{x}{2}} u_{N}$ be the approximation of $v$, the solution of the original Burgers equation (4.1). Similarly to the proof of Theorem 3.3, we can establish the following result:

Theorem 4.3 Let s, $r \geq 1, \alpha$ and $\beta$, are the largest integers for which $\alpha<s$ and $\beta<r$. Then, for all $t \in(0, T]$,

$$
\begin{gathered}
\left\|v_{N}(t)-v(t)\right\|_{1} \\
(4.15) \leq C_{1}\left(N^{\frac{1}{2}-\frac{s}{2}}\|v\|_{L^{\infty}\left(0, T ; H_{\alpha}^{s}\left(\mathbb{R}_{+}\right)\right)}+N^{\frac{1}{2}-\frac{r}{2}}\left\|v_{t}\right\|_{L^{2}\left(0, T ; H_{\beta}^{r}\left(\mathbb{R}_{+}\right)\right)}\right) .
\end{gathered}
$$

Remark 4.1 The result in Theorem 4.3 indicates that the Laguerre-Galerkin approximation may converge exponentially, even if $v$ only delays algebraicly at infinity, as long as the norms on the right-hand side of (4.15) are bounded for all $s, r>0$.

\section{Efficient implementations and numerical results}

We consider first the Laguerre-approximation of the transformed Burgers equation (4.7). After discretizing it in time by using a semi-implicit scheme, i.e. the linear term is treated implicitly while the nonlinear term is treated explicitly, we need to solve, at each time step, a linear system of the form: find $w_{N} \in \mathbb{P}_{N}^{0}$ such that

$$
\alpha_{1}\left(\frac{d w_{N}}{d x}, \frac{d v}{d x}\right)_{\omega}+\alpha_{2}\left(w_{N}, v\right)_{\omega}=(g, v)_{\omega}, \quad \forall v \in \mathbb{P}_{N}^{0} .
$$

As in $[17,18]$, the efficiency of the spectral-Galerkin method depends heavily on the choice of a suitable basis. We present an efficient implementation for (5.1) below.

Let $\phi_{i}(x)=L_{i}(x)-L_{i+1}(x)$. Since $L_{i}(0)=1$ for all $i$, we have $\phi_{i}(0)=0$ which implies that

$$
\mathbb{P}_{N}^{0}=\operatorname{span}\left\{\phi_{0}, \phi_{i}, \ldots, \phi_{N-1}\right\} .
$$


Furthermore, let $v^{\prime}(x)=\frac{d v}{d x}(x)$. We have

$$
\phi_{i}^{\prime}(x)=L_{i}^{\prime}(x)-L_{i+1}^{\prime}(x)=L_{i}(x) .
$$

Therefore,

$$
a\left(\phi_{i}, \phi_{j}\right)=\left(\phi_{i}^{\prime}, \phi_{j}^{\prime}\right)_{\omega}=\left(L_{i}^{\prime}, L_{j}^{\prime}\right)_{\omega}=\delta_{i j} .
$$

In other words, $\left\{\phi_{i}\right\}_{i=0,1, \ldots, N-1}$ form an orthonormal basis of $\mathbb{P}_{N}^{0}$ with respect to the inner product $a(\cdot, \cdot)$. We can then look for $w_{N}$ in the form of $u_{N}=\sum_{i=0}^{N-1} h_{N, i} \phi_{i}$. Let us denote

$$
\begin{aligned}
& \boldsymbol{h}=\left(h_{N, 0}, h_{N, 1}, \ldots, h_{N, N-1}\right)^{\mathrm{T}}, \\
& g_{i}=\left(g, \phi_{i}\right)_{\omega}, \quad \boldsymbol{g}=\left(g_{0}, g_{1}, \ldots, g_{N-1}\right)^{\mathrm{T}}, \\
& c_{i j}=\left(\phi_{j}, \phi_{i}\right), \quad C=\left(c_{i j}\right)_{i, j=0,1, \ldots, N-1} .
\end{aligned}
$$

Then, (5.1) is reduced to the following linear system:

$$
\left(\alpha_{1} I+\alpha_{2} C\right) \boldsymbol{h}=\boldsymbol{g} .
$$

Thanks to (2.2) and (5.2), we find

$$
c_{i j}= \begin{cases}2, & i=j \\ -1, & i=j-1, j+1 . \\ 0, & \text { otherwise }\end{cases}
$$

Hence (5.5) is a linear symmetric tridiagonal system which can be easily solved. Note that the Laguerre-Galerkin method above does not suffer from the ill-conditioning usually related to Laguerre-collocation/tau methods.

We now consider the Laguerre-Galerkin approximation for the transformed BBM equation (3.7). Using the same notations as above, and denoting additionally

$$
\begin{aligned}
u_{N}(x, t) & =\sum_{i=0}^{N-1} h_{N, i}(t) \phi_{i}(x), \\
H_{N}(t) & =\left(h_{N, 0}(t), h_{N, 1}(t), \ldots, h_{N, N-1}(t)\right)^{\mathrm{T}}, \\
P_{N}^{1,0} u_{0}(x) & =\sum_{i=0}^{N-1} h_{N, i}^{0} \phi_{i}(x), \quad H_{N}^{0}=\left(h_{N, 0}^{0}, h_{N, 1}^{0}, \ldots, h_{N, N-1}^{0}\right)^{\mathrm{T}},
\end{aligned}
$$

and

$$
\begin{aligned}
G_{i}\left(H_{N}, t\right) & =\left(f, \phi_{i}\right)_{\omega}-b\left(u_{N}, u_{N}, \phi_{i}\right), \\
G\left(H_{N}, t\right) & =\left(G_{0}\left(H_{N}, t\right), G_{1}\left(H_{N}, t\right), \ldots, G_{N-1}\left(H_{N}, t\right)\right)^{\mathrm{T}},
\end{aligned}
$$



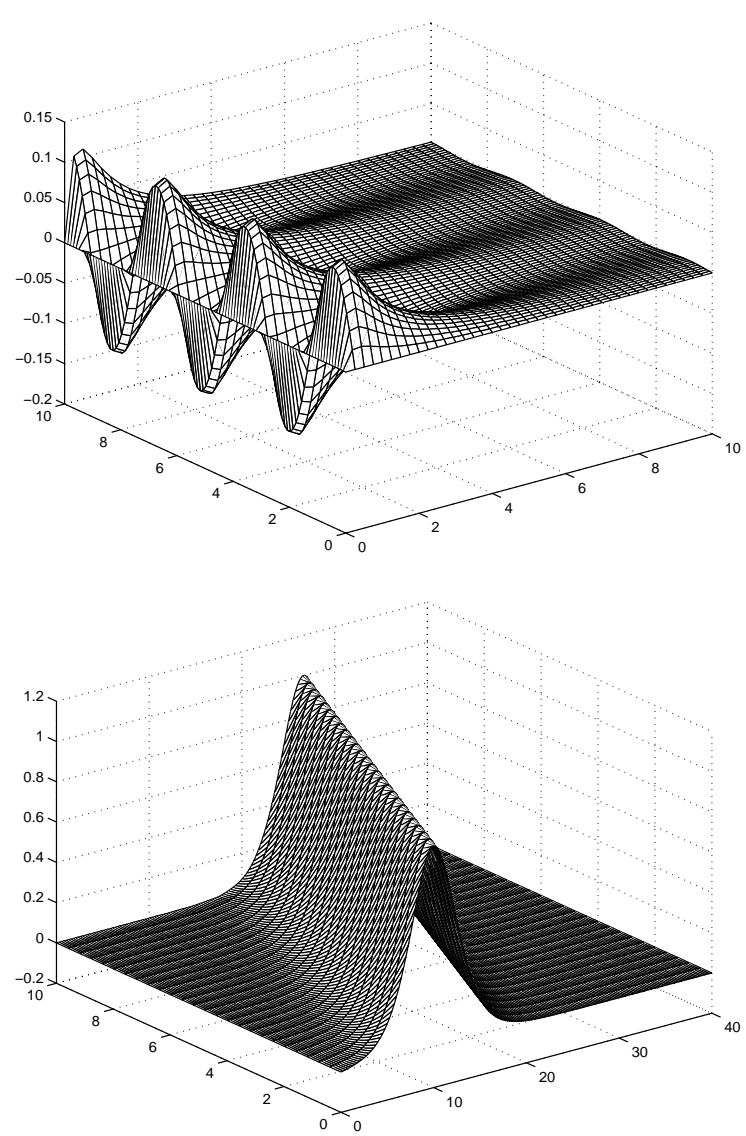

Fig. 1. Exact and approximation solutions: Example 1 with $k=2$ and $h=3.5$ is on the left, Example 2 is on the right

we find that, thanks to (5.3), (3.7) becomes the following system of ordinary differential equations:

$$
\frac{d}{d t} H_{N}(t)=G\left(H_{N}, t\right), \quad H_{N}(0)=H_{N}^{0}
$$

We can then apply a standard explicit Runge-Kutta method to the above system without suffering from stringent time step constraint.

We now present some numerical results. We discretize the transformed Burgers equation (4.2) by a third-order BDF scheme for the linear terms and third-order Adams-Bashforth scheme for the nonlinear term; for the transformed BBM equation (3.3), a standard forth-order Runge-Kutta method is used for (5.9). To illustrate the spectral accuracy, the time step is chosen to be sufficiently small so that the error is dominated by the spatial discretization 

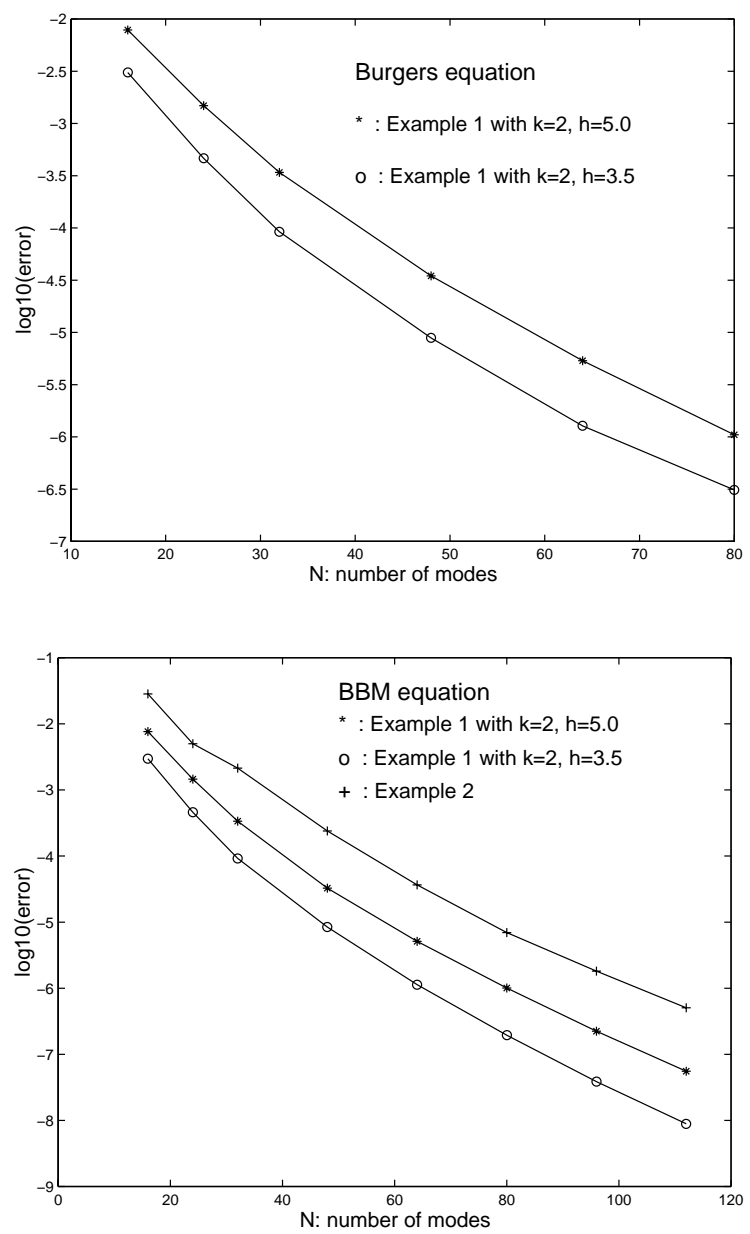

Fig. 2. Maximum Error at $t=1$ of the Laguerre-Galerkin approximation for the Burgers and BBM equations

error. We consider the following two examples where one solution decays algebraicly at infinity while the other decays exponentially at infinity.

Example 1. The exact solution of the original Burgers equation (4.1) and BBM equation (3.1) being $v(x, t)=\frac{\sin k t}{(1+x)^{h}}$. (algebraic decay at infinity)

Example 2. The exact solution of the original BBM equation (3.1) being a solitary wave $v(x, t)=\operatorname{sech}^{2}(a x-b t-c)$ with $a=.3, b=.5$ and $c=3$. (exponential decay at infinity)

In Fig. 1, we plot the exact and approximate solutions for the original Burgers and BBM equations obtained with $N=64$ in the space-time domain 
$[0,10] \times[0,10]$. The exact and approximate solutions are indistinguishable in both cases.

To illustrate the quantitative behaviors of the Laguerre-Galerkin approximations, we plot in Fig. $2 \log _{10}\left(\left\|v_{N}-v\right\|_{L^{\infty}\left(\mathbb{R}_{+}\right)}\right)$vs. $N$ at $t=1$ for the original Burgers and BBM equations. Note that exponentially convergence for the original Burgers and BBM equations is achieved in all cases regardless whether the exact solution decays exponentially or algebraicly at infinity.

\section{Concluding remarks}

We have presented an efficient and accurate Laguerre-Galerkin method for the Burgers and BBM equations on a semi-infinite interval. We used a suitable functional transform which not only removes growing numerical errors for $x$ large but also makes the transformed equations well-posed with a weighted variational formulation. Our theoretical and numerical results shown that the Laguerre-Galerkin approximations are stable and convergent on a semi-infinite interval with spectral accuracy. Furthermore, the Laguerre-Galerkin approximation may converge exponentially, even if the solution $v$ only delays algebraicly at infinity, as long as $\|u(t)\|_{r, \beta}$ are finite for all $r>0$.

\section{References}

1. Kh. O. Abdulloev, I. L. Bogolubsky, and V. G. Makhankov. One more example of inelastic solution interaction. Phys. Rev. Lett. A, 56:427-428,1976

2. R. A. Adams. Sobolev Spaces. Acadmic Press, New York, 1975

3. M. E. Alexander and J. L. Morris. Galerkin methods applied to some model equation for nonlinear dispertive waves. J. Comp. Phys., 30:428-451,1979

4. T. B. Benjamin, J. L. Bona, and J. J. Mahony. Model equation for long waves in nonlinear dispertive systems. Phil. Trans. R. Soc. Lond. A, 272:47-48,1972

5. C. Bernardi and Y. Maday. Spectral method. In P. G. Ciarlet and L. L. Lions, editors, Handbook of Numerical Analysis, V. 5 (Part 2). North-Holland,1997

6. J. L. Bona and P. J. Bryant. A mathematical model for long waves generated by wavemakers in nonlinear dispersive systems. Proc. Camb. Phil. Soc., 73:391,1973

7. J. L. Bona, W. G. Pritchard, and L. R. Scott. An evaluation of a model equation for water waves. Phil. Trans. R. Soc. Lond. A, 302:457-510,1981

8. C. Canuto, M. Y. Hussaini, A. Quarteroni, and T. A. Zang. Spectral Methods in Fluid Dynamics. Springer-Verlag, Berlin, 1987

9. O. Coulaud, D. Funaro, and O. Kavian. Laguerre spectral approximation of elliptic problems in exterior domains. Comp. Mech. in Appl. Mech and Eng., 80:451-458,1990

10. D. Funaro. Estimates of Laguerre spectral projectors in sobolev spaces. In C. Brezinski,

L. Gori, and A. Ronveaux, editors, Orthogonal Polynomials and their Applications, pages 263-266. Scientific Publishing, 1991 
11. D. Gottlieb and S. A. Orszag. Numerical Analysis of Spectral Methods: Theory and Applications. SIAM-CBMS, Philadelphia,1977

12. Benyu Guo. A class of difference schemes of two-dimensional viscous fluid flow. Acta Math. Sinica, 17:242-258,1974

13. Benyu Guo. Finite Difference Methods for Partial Differential Equations. Science Press, Beijing, 1988

14. V. Iranzo and A. Falqués. Some spectral approximations for differential equations in unbounded domains. Comp. Mech. in Appl. Mech. and Eng., 98:105-126,1992

15. Y. Maday, B. Pernaud-Thomas, and H. Vandeven. Une réhabilitation des méthodes spèctrales de type laguerre. Rech. Aerospat., 6:353-379,1985

16. C. Mavriplis. Laguerre polynomails for infinite-domain spectral elements. J. Comp. Phys., 80:480-488,1989

17. Jie Shen. Efficient spectral-Galerkin method I. direct solvers for second- and fourthorder equations by using Legendre polynomials. SIAM J. Sci. Comput., 15:14891505,1994

18. Jie Shen. Efficient spectral-Galerkin method II. direct solvers for second- and fourthorder equations by using Chebyshev polynomials. SIAM J. Sci. Comput., 16:7487,1995

19. H. J. Stetter. Stabilitiy of nonlinear discretization algorithms. In J. Bramble, editor, Numerical Solutions of Partial Differential Equations, pages 111-123. Academic Press, New York,1966 